\title{
Aprendiendo en base a problemas y trabajo por proyectos de investigación en Ciencias Sociales
}

\author{
JORGE RUIZ MORALES \\ Universidad de Sevilla \\ Dpto. Didáctica de las Ciencias \\ Experimentales y Sociales \\ jruiz2@us.es \\ ORCID: http://orcid.org/0000-0002-6081-9363 \\ D.O.I.: http://dx.doi.org/10.12795/JDU.2018.i01.82 \\ Pp.: 1460-1477
}

\section{Resumen}

Esta comunicación nos aproxima al tercer ciclo de mejora con estudiantes de tercer curso del Grado de Educación Primaria, en la asignatura de Didáctica de las Ciencias Sociales, Facultad de Ciencias de la Educación. En esta ocasión siguiendo con la idea de promover un espacio seguro de aprendizaje autónomo, se ha optado por indagar cómo implementar desde una perspectiva más práctica la aplicación de los conocimientos de la unidad 2 y 3 de la asignatura.

Se ha buscado aprovechar el aprendizaje en base a problemas y el trabajo por proyectos de investigación en Ciencias Sociales, investigando de qué modo se puede mejorar la implementación de los contenidos teóricos con la realización de actividades en el aula, aprender a gestionar el trabajo grupal y poner de relieve los procesos de participación 
ciudadana y el abordaje de los problemas socioambientales en las ciencias sociales.

Palabras Clave: Didáctica de las ciencias sociales, educación primaria, docencia universitaria, experimentación docente universitaria, innovación.

\section{Breve descripción del contexto.}

El modelo didáctico en construcción (MDC) que año a año va tomando sentido desde la realidad del aula, posee algunas ideas claves, desde las que vengo trabajando, fruto de la inquietud como docente por seguir aprendiendo, mejorar, facilitar procesos de aprendizaje experienciales, vivir la construcción del conocimiento de modo personal y colectivo en el grupo-clase, etc.

Esto está lógicamente influenciado por factores contextuales, la asignatura de didáctica de las ciencias sociales es cuatrimestral, hecho que marca el proceso de aprendizaje, porque año tras año el proyecto docente comprobamos que está diseñado para una asignatura anual; dicho de otro modo, si queremos lograr lo que se plantea como objetivos, competencias y contenidos, deberíamos tener al menos un año o una asignatura perfectamente sincronizada que se trabajase en dos cuatrimestres. Este año en la asignatura, después de once semanas, hemos compartido la asignatura tres profesores, ha habido una baja por paternidad y un puente.

Además, cada año en la asignatura de Didáctica de las Ciencias Sociales hay un grupo distinto, por tanto lo que parece funcionar bien con un grupo, no tiene por qué servir para otro. Incluso yo no soy el mismo, por tanto hay cuestiones personales y profesionales que afectan a mi propio modo de entender la docencia, el trabajo que se realiza en el aula, la relación con los estudiantes, etc. Por último, las personas que nos encontramos en una situación de precariedad laboral trabajando en la Universidad, que se prolonga en el tiempo, viviendo situaciones de incertidumbre que a veces se cronifican, nos afecta sobremanera a la hora de enfocar nuestro trabajo. Todo ello en 
un contexto universitario y socioeconómico, donde aparece la capitalización del conocimiento científico, o lo que se ha dado en llamar capital académico, según Barchiesi y otros (2010). Porque todo ello está modificando el modo en el que nos sentimos, nos configuramos y nos comportamos en los diferentes espacios universitarios.

Los avances, estancamientos y procesos en el MDC están afectados por cuestiones estructurales que forjan contextos que influyen sobre la actividad docente, la investigación, las relaciones y la comunidad universitaria.

Los elementos claves sobre los que se asienta mi MDC aparecen en la figura 1, se han configurado fruto de mi implicación en procesos de participación y transformación social; la formación académica, licenciado en Pedagogía, la experiencia profesional fuera de la Universidad, de más de 22 años; la trayectoria investigadora en el campo social, educativo y universitario, alrededor de 20 años; y el análisis de mi práctica docente en las asignaturas de Didáctica de las Ciencias Sociales, Formación Inicial de Maestros/as y Trabajos Fin de Estudios (Ruiz-Morales, 2017a, 2017b), además del trabajo desempeñado en la actualidad en la UNIR en formación a distancia.

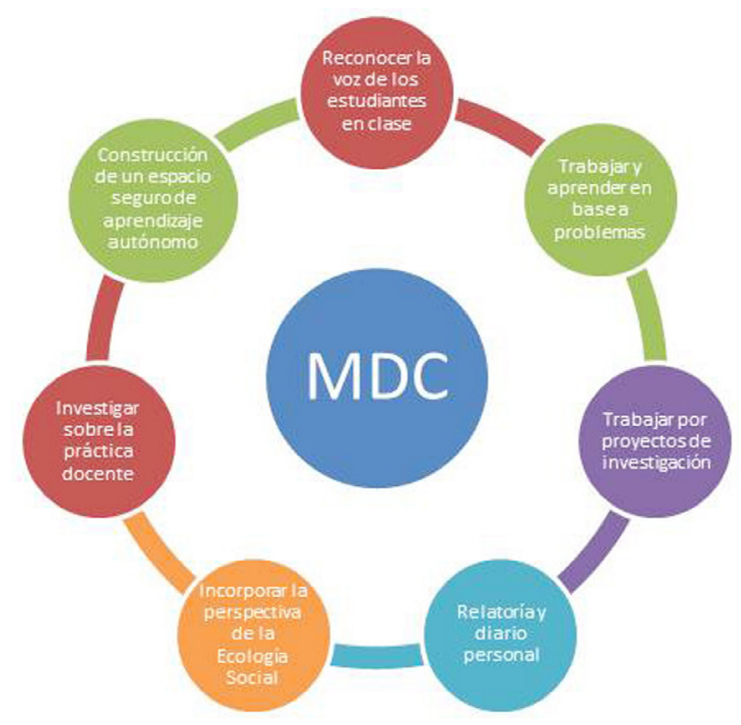

Figura 1. Elementos identificativos del MDC

Jornadas de Formación e Innovación Docente del Profesorado I № 1 (2018) Esta obra se distribuye con la licencia Creative Commons 
En nuestro papel como formador de formadores, puesto que trabajamos con maestros/as y profesorado en formación inicial, tiene una fuerte influencia la cosmovisión que sobre la educación y la sociedad vamos configurando, fruto de nuestras experiencias, procesos de crianza, participación en comunidades educativas, iniciativas y proyectos de innovación.

\section{Diseño previo del Ciclo de Mejora Docente}

El mapa de conocimientos deseable para construir un proceso de aprendizaje dialógico con los estudiantes se basa en los problemas que se presentaron con anterioridad a desarrollar la programación de actividades en el aula, como parte del pre-diseño que se compartió en el grupo de trabajo de la REFID.

Los problemas sobre los que he centrado mi tercer ciclo de mejora docente (CMD) son los siguientes:

- ¿Cómo mejorar la implementación de los contenidos teóricos con la realización de actividades en el aula?

- ¿Aprendemos investigando sobre problemas socioambientales?

- ¿De qué modo aprendemos a planificar y gestionar los tiempos para ser más eficientes en nuestra actividad profesional?

- ¿Qué importancia tienen los procesos de participación ciudadana y el abordaje de los problemas socioambientales en las ciencias sociales?

Los problemas de investigación van tomando cuerpo en forma de conocimientos sobre los que hemos trabajado en este ciclo de mejora, estos están relacionados con objetivos, contenidos, estrategias metodológicas y actitudes, que se representan en la figura 2. 


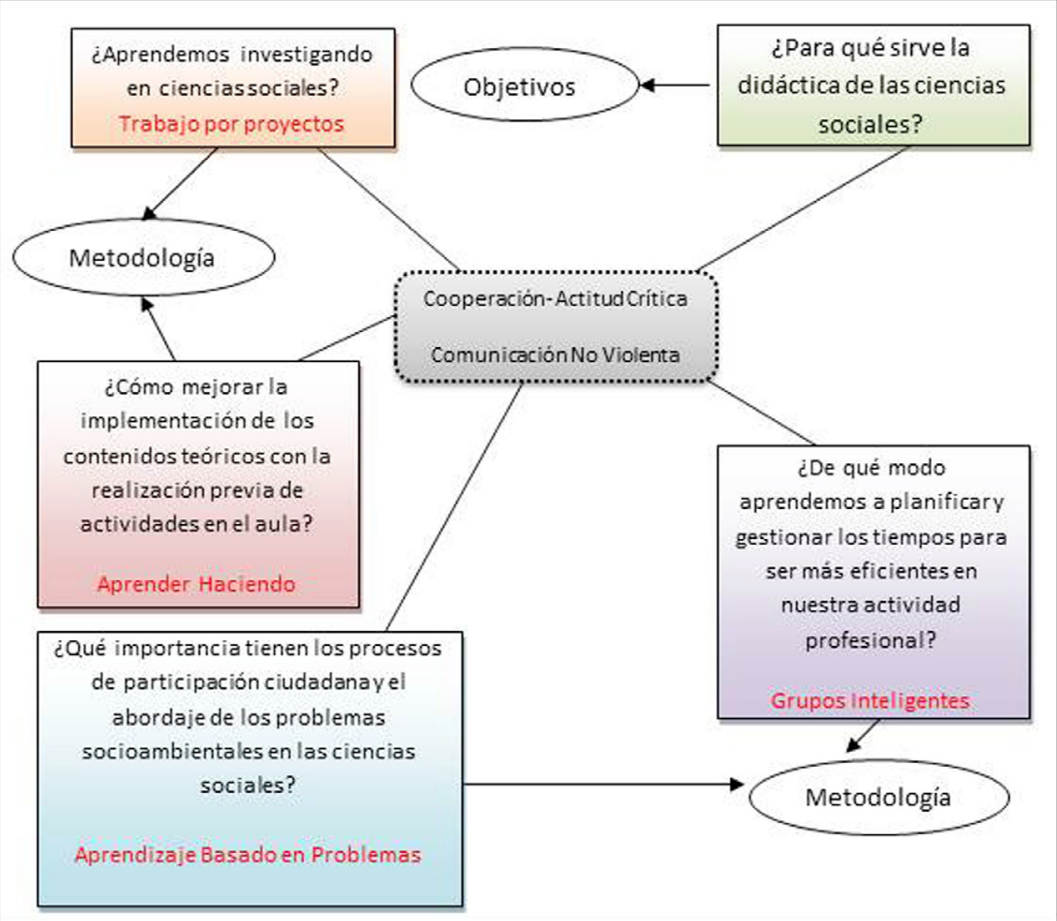

Figura 2. Trama de conocimientos y contenidos del ciclo de mejora

\section{Modelo metodológico y secuencia de actividades}

El modelo metodológico posible está basado en lo presentado en la figura 1, además se presenta la secuencia didáctica, que de algún modo organiza el conjunto de la asignatura (ver figura 3) para tener una visión global de la misma, y por último la planificación de actividades desarrolladas que aparece a continuación en la figura 4 (Ruiz-Morales y Ferreras, 2018, pág.462.), que ha tenido alguna modificación fruto de la propia práctica docente y de las dificultades acaecidas este trimestre en las sesiones de prácticas.

Jornadas de Formación e Innovación Docente del Profesorado I № 1 (2018) Esta obra se distribuye con la licencia Creative Commons Reconocimiento-NoComercial-SinObraDerivada Internacional (CC BY-NC-ND 4.0.) 


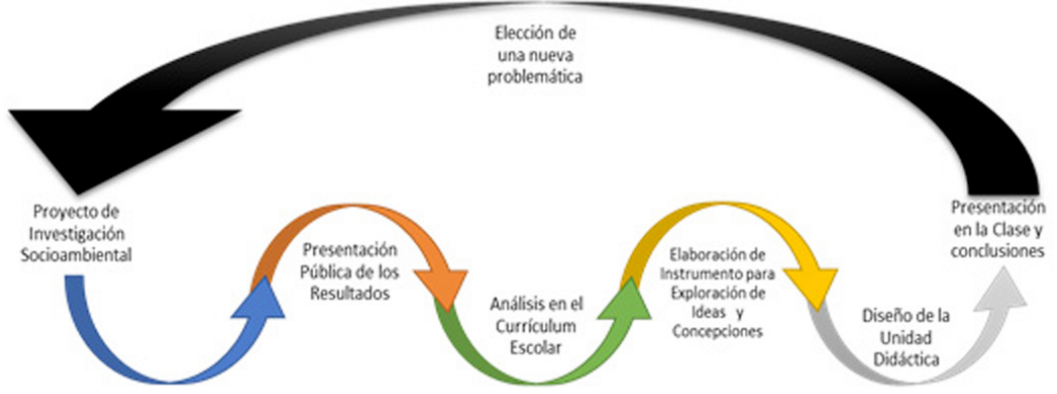

Figura 3. Secuencia didáctica del modelo didáctico personal

La secuencia didáctica que se sigue en la asignatura busca que los estudiantes vayan construyendo un modelo didáctico ajustado a sus objetivos, expectativas, metodología, que posea una coherencia interna, que se pueda ajustar a trabajar con las ideas, intereses, propuestas y necesidades del alumnado de primaria. Por ello en las sesiones se intenta reflexionar sobre el modo en el que se trabajan los contenidos en la asignatura y cómo ellos se imaginan trabajar en sus clases, esto va tomando cuerpo en el diario personal que entregan a final de cuatrimestre.

Las sesiones de clase parten siempre de una relatoría que los estudiantes elaboran del día anterior, de modo que la palabra de los estudiantes se hace patente desde el principio de la sesión. A continuación solemos dedicar algún tiempo para hablar de algunas cuestiones de procedimiento o alguna información de interés sobre algún acontecimiento, evento o algo que puede ser significativo para la asignatura. Solemos dejar parte de las sesiones para actividades grupales relacionadas con los contenidos de la asignatura, que permite que el profesor pueda interactuar de modo más próximo con cada grupo de trabajo, que se encarga del proyecto de investigación, la realización del póster o dossier, de la lectura de los artículos, etc.

Son muy importantes las puestas en común que realizamos en la clase, porque permite reflexionar como grupo, 

cimientos, se facilita que los estudiantes más retraídos se lancen a hablar en el grupo grande, se tomen en consideración todas las perspectivas y se produzca un pensamiento colectivo.

Por último tienen gran relevancia las actividades que hacemos fuera del aula (parque junto a la facultad, Ecomuseo Miraflores, investigación social, etc.), porque les permite comprobar que aprendemos en múltiples espacios, genera cambios en las energías del grupo y facilita las interacciones.

\begin{tabular}{|c|c|c|c|}
\hline $\begin{array}{l}\text { Unidades } \\
\text { Didácticas }\end{array}$ & Sesiones & Actividades & Temporalización \\
\hline \multirow{17}{*}{ Unidad 1} & \multirow[b]{3}{*}{$24 / 9 / 2018$} & $\begin{array}{l}\text { Presentación del programa, trama de conocimientos, } \\
\text { trama de contenidos y sistema de evaluación }\end{array}$ & $40^{\prime}$ \\
\hline & & Diario personal y cuaderno de bitácoras. & $20^{\prime}$ \\
\hline & & Cierre de la sesión. & 5 \\
\hline & \multirow{5}{*}{$25 / 9 / 2018$} & Relatoría del grupo-clase. & $10^{\prime}$ \\
\hline & & Actividad 1.1. Cuestionario de ideas previas & $20^{\prime}$ \\
\hline & & $\begin{array}{l}\text { ¿Cómo está organizada la información en la } \\
\text { plataforma de enseñanza virtual de la US? }\end{array}$ & $15^{\prime}$ \\
\hline & & $\begin{array}{l}\text { Presentación de los miembros del grupo: } \\
\text { - Técnica de presentación por parejas en } \\
\text { bandos. } \\
\text { - Los bloques. } \\
\text { - La estrella. }\end{array}$ & 30 \\
\hline & & Cierre de la sesión. & 5 \\
\hline & \multirow[b]{4}{*}{$1 / 10 / 2018$} & Relatoría del grupo-clase. & $5^{\prime}-10^{\prime}$ \\
\hline & & $\begin{array}{l}\text { ¿Cómo entendemos el proceso de enseñanza- } \\
\text { aprendizaje en la asignatura? }\end{array}$ & $30^{\prime}$ \\
\hline & & ¿Cómo investigamos en ciencias sociales? & $35^{\prime}$ \\
\hline & & Cierre de la sesión. & 5 \\
\hline & \multirow{4}{*}{$\begin{array}{c}4 \\
2 / 10 / 2018\end{array}$} & Relatoría del grupo-clase. & $5^{\prime}-10^{\prime}$ \\
\hline & & $\begin{array}{l}\text { Conformación de los grupos, elección de las } \\
\text { problemáticas socioambientales vinculándolas con } \\
\text { el territorio y procesos de participación ciudadana. }\end{array}$ & $40^{\prime}$ \\
\hline & & Debate sobre los resultados del cuestionario inicial & $20^{\prime}$ \\
\hline & & Cierre de la sesión. & 5 \\
\hline & $\begin{array}{c}5 \\
5 / 10 / 2018\end{array}$ & $\begin{array}{l}\text { Elaboración de pre-diseño del proyecto de } \\
\text { investigación y ficha de grupo. }\end{array}$ & $80^{\prime}$ \\
\hline
\end{tabular}

Jornadas de Formación e Innovación Docente del Profesorado | № 1 (2018) Esta obra se distribuye con la licencia Creative Commons Reconocimiento-NoComercial-SinObraDerivada Internacional (CC BY-NC-ND 4.0.) 


\begin{tabular}{|c|c|c|c|}
\hline $\begin{array}{l}\text { Unidades } \\
\text { Didácticas }\end{array}$ & Sesiones & Actividades & Temporalización \\
\hline \multirow[t]{14}{*}{ Unidad 2} & \multirow{5}{*}{$8 / 10 / 2018$} & Relatoría del grupo-clase. & $5^{\prime}-10^{\prime}$ \\
\hline & & Aprobación del programa de la asignatura & $30^{\prime}$ \\
\hline & & $\begin{array}{l}\text { Actividad } 2.1 \text { Aprendiendo a trabajar con } \\
\text { problemas socioambientales. Análisis de conflictos } \\
\text { y aportaciones del conocimiento científico de las } \\
\text { ciencias sociales. }\end{array}$ & $20^{\prime}$ \\
\hline & & Cuestiones organizativas de la asignatura. & $15^{\prime}$ \\
\hline & & Cierre de la sesión. & $5^{\prime}$ \\
\hline & \multirow{4}{*}{$\begin{array}{c}7 \\
9 / 10 / 2018\end{array}$} & Relatoría del grupo-clase. & $10^{\prime}$ \\
\hline & & $\begin{array}{l}\text { Actividad } 2.1 \text { Aprendiendo a trabajar con } \\
\text { problemas socioambientales. }\end{array}$ & $30^{\prime}$ \\
\hline & & Proyecto de Investigación & $30^{\prime}$ \\
\hline & & Cierre de la sesión. Eventos de interés. & $10^{\prime}$ \\
\hline & \multicolumn{3}{|c|}{ INICIO CICLO DE MEJORA 2018} \\
\hline & \multirow{4}{*}{$15 / 10 / 2018$} & Relatoría del grupo-clase. & $5^{\prime}-10^{\prime}$ \\
\hline & & Proyecto de Investigación & $30^{\prime}$ \\
\hline & & $\begin{array}{l}\text { ¿Es posible otra enseñanza de las ciencias sociales? } \\
\text { Lectura colectiva de artículos de la unidad. }\end{array}$ & $35^{\prime}$ \\
\hline & & Cierre de la sesión. & 5 \\
\hline \multicolumn{4}{|l|}{ Didácticas } \\
\hline & & $\begin{array}{l}\text { Cierre de la sesión. ¿Qué importancia tienen los } \\
\text { procesos de participación ciudadana con el abordaje } \\
\text { de los problemas socioambientales? }\end{array}$ & $5^{3}$ \\
\hline & \multirow[t]{2}{*}{15} & Relatoría del grupo-clase & $10^{\prime}$ \\
\hline & & $\begin{array}{l}\text { Educaciones centrifugas a cargo de Javier Encina y } \\
\text { Ainhoa Ezeiza }\end{array}$ & $70^{\prime}$ \\
\hline & $\begin{array}{c}16 \\
2 / 11 / 2018\end{array}$ & $\begin{array}{l}\text { ¿Cuáles son las ideas y concepciones del alumnado } \\
\text { sobre nuestras problemáticas socioambientales? } \\
\text { Elaboración de instrumento }\end{array}$ & $80^{\prime}$ \\
\hline & \multirow{4}{*}{$\begin{array}{c}17 \\
5 / 11 / 2018 \\
\text { (primera } \\
\text { entrega del } \\
\text { diario } \\
\text { personal) }\end{array}$} & Relatoría del grupo-clase. & $5^{\prime}-10^{\prime}$ \\
\hline & & $\begin{array}{l}\text { Preparación de Actividad en el Parque Nacional de } \\
\text { Monfragüe }\end{array}$ & $30^{\prime}$ \\
\hline & & $\begin{array}{l}\text { Elaboración de póster y dossier de la investigación } \\
\text { realizada. }\end{array}$ & $35^{\prime}$ \\
\hline & & Cierre de la sesión. & 5 \\
\hline & \multirow{4}{*}{$\begin{array}{c}18 \\
6 / 11 / 2018\end{array}$} & Relatoría del grupo-clase. & $5^{\prime}-10^{\prime}$ \\
\hline & & $\begin{array}{l}\text { ¿Cuáles son las ideas y concepciones del alumnado } \\
\text { sobre nuestras problemáticas socioambientales? }\end{array}$ & $50^{\prime}$ \\
\hline & & $\begin{array}{l}\text { Preparación de Actividad en el Parque Nacional de } \\
\text { Monfrágüe }\end{array}$ & $20^{\prime}$ \\
\hline & & Cierre de la sesión. & 5 \\
\hline & $\begin{array}{c}\text { Tutoria } \\
\text { grupal } \\
9 y \\
10 / 1 / 2018\end{array}$ & $\begin{array}{l}\text { Cada grupo es interrogado por qué ha aprendido en } \\
\text { el proyecto de investigación, en el trabajo en grupo } \\
\text { y se revisa el instrumento para el análisis de ideas y } \\
\text { concepciones. }\end{array}$ & $30^{\prime}$ por grupo \\
\hline & \multirow{2}{*}{$\begin{array}{c}19 \\
9 / 11 / 2018\end{array}$} & Relatoría & 5 \\
\hline & & $\begin{array}{l}\text { ¿Cuáles son las ideas y concepciones del alumnado } \\
\text { sobre nuestras problemáticas socioambientales? }\end{array}$ & $75^{\prime}$ \\
\hline & \multirow{3}{*}{$\begin{array}{c}20 \\
12 / 11 / 2018\end{array}$} & Relatoría del grupo-clase. & $10^{\prime}$ \\
\hline & & Revisión de los póster y diarios personales & $70^{\prime}$ \\
\hline & & Cierre de la sesión. & $10^{\prime}$ \\
\hline
\end{tabular}




\begin{tabular}{|c|c|c|c|}
\hline & \multirow{5}{*}{$\begin{array}{c}21 \\
13 / 11 / 2018\end{array}$} & Relatoría & \multirow{2}{*}{$\frac{10^{3}}{10^{3}}$} \\
\hline & & $\begin{array}{l}\text { Presentación de Laboratorio } \\
\text { Patrimonio y Territorio. CIPAT }\end{array}$ & \\
\hline & & Presentación de la Propuesta Didáctica & $30^{\prime}$ \\
\hline & & Síntesis de ideas clave sobre la unidad 2 & $20^{2}$ \\
\hline & & Cierre de sesión & $10^{\prime}$ \\
\hline & \multirow[b]{2}{*}{$16 / 11 / 2018$} & Relatoría & $5^{\prime}$ \\
\hline & & $\begin{array}{l}\text { ¿Cuáles son las ideas y concepciones del alumnado } \\
\text { sobre nuestras problemáticas socioambientales? } \\
\text { Finalización del informe }\end{array}$ & \\
\hline \multirow[t]{15}{*}{$\begin{array}{l}\text { Unidades } \\
\text { Didácticas }\end{array}$} & Sesiones & Actividades & Temporalización \\
\hline & \multirow[b]{3}{*}{$16 / 10 / 2018$} & Relatoria del grupo-clase. & $5^{\prime}-10^{\prime}$ \\
\hline & & Proyecto de investigación. Elaboración del Póster. & $30^{\prime}$ \\
\hline & & Cierre de la sesión. & 5 \\
\hline & \multirow{2}{*}{10} & Relatoría del grupo-clase. & $5^{\prime}-10^{\prime}$ \\
\hline & & Proyecto de Investigación. Entrega el 28/10/2018 & $70^{3}$ \\
\hline & \multicolumn{2}{|c|}{ Tutoría grupal para seguimiento de la asignatura } & $30^{\prime}$ con cada grupo \\
\hline & \multirow{7}{*}{$22 / 10 / 2018$} & Relatoría del grupo-clase. & $5^{\prime}-10^{\prime}$ \\
\hline & & $\begin{array}{l}\text { Presentación del Itinerario Interpretativo por el } \\
\text { Ecomuseo Miraflores. Actividad 2.2. Entrega el } \\
4 / 11 / 2018\end{array}$ & $30^{\prime}$ \\
\hline & & $\begin{array}{l}\text { Doc. 2.8., 2.1., 2.3, 2.6., también artículo de la } \\
\text { sección de la plataforma: metodología de la } \\
\text { asignatura. Problemas del mundo y educación } \\
\text { escolar: un desafío para la enseñanza de la geografía } \\
\text { y las Ciencias Sociales. Trabajo colectivo sobre } \\
\text { artículos de la unidad en base a transparencia }\end{array}$ & $30^{\prime}$ \\
\hline & & $\begin{array}{l}\square \text { CORRECCIÓN de errores de la Orden de } 17 \text { de } \\
\text { marzo de } 2015 \text {, por la que se desarrolla el currículo } \\
\text { correspondiente a la Educación Primaria en } \\
\text { Andalucía (BOJA 29-10-2015). }\end{array}$ & \\
\hline & & $\begin{array}{l}\square \text { ORDEN de } 17 \text { de marzo de } 2015 \text {, por la que se } \\
\text { desarrolla el currículo correspondiente a la } \\
\text { Educación Primaria en Andalucía (BOJA 27-03- } \\
\text { 2015). (19,7 MB) }\end{array}$ & \\
\hline & & $\begin{array}{l}\text { DECRETO 97/2015, de } 3 \text { de marzo, por el que se } \\
\text { establece la ordenación y el currículo de la } \\
\text { Educación Primaria en la Comunidad Autónoma de } \\
\text { Andalucía (BOJA 13-03-2015). }\end{array}$ & \\
\hline & & $\begin{array}{l}\text { Cierre de la sesión. Recordar Entrega del Póster y } \\
\text { Dossier } 5 / 11 / 2018\end{array}$ & 5 \\
\hline & $\begin{array}{c}12 \\
24 / 10 / 2018\end{array}$ & $\begin{array}{l}\text { Interpretación por el Ecomuseo Miraflores. } \\
\text { Ejemplificación de realidad aumentada. } \\
\text { Rol-playin. }\end{array}$ & $120^{\prime}$ \\
\hline
\end{tabular}




\begin{tabular}{|c|l|c|}
\hline \multirow{3}{*}{$26 / 10 / 2018$} & $5^{\prime}-10^{\prime}$ \\
\cline { 2 - 3 } & $\begin{array}{l}\text { Relatoría del grupo-clase } \\
\text { ¿Cuáles son las ideas y concepciones del alumnado } \\
\text { sobre nuestras problemáticas socioambientales? } \\
\text { Elaboración de instrumento }\end{array}$ & $30^{\prime}$ \\
\cline { 2 - 3 } & $\begin{array}{l}\text { Aproximación al curriculum escolar } \\
\text { Cierre de la sesión. }\end{array}$ & $30^{\prime}$ \\
\hline 14 & \multicolumn{1}{|c|}{ Relatoría del grupo-clase. } & $5^{\prime}-10^{\prime}$ \\
\cline { 2 - 3 } & $\begin{array}{l}\text { Presentación de contenidos Doc unidad 2 y } \\
\text { legislación, entrega 11/11/2018 }\end{array}$ & \\
\hline & $\begin{array}{l}\text { Presentación de informe investigación sobre ideas y } \\
\text { concepciones del alumnado 18/11/2018 }\end{array}$ & \\
\hline & $\begin{array}{l}\text { Informe Miraflores presentación 7/11/2018 } \\
\text { Presentación de la experiencia de la investigación y } \\
\text { propuesta didáctica elaborada para Miraflores en } \\
\text { relación con los movimientos sociales por Adrián } \\
\text { Domínguez Domínguez. }\end{array}$ & \\
\hline
\end{tabular}

Figura 4. Programación de actividades del tercer ciclo de mejora

\section{Aplicación del ciclo de mejora}

Las sesiones han ido transcurriendo introduciendo algunos cambios entre la programación de actividades entregada en la propuesta antes de empezar, y la que se muestra en la figura 4.

En las primeras sesiones dedicamos algún tiempo para presentar la asignatura, invitar a los estudiantes a leer el programa y proponer propuestas desde sus intereses y necesidades. Es importante resaltar que como años anteriores, se sorprenden de que se les invite a participar en la elaboración y aprobación del programa de la asignatura. Como siempre llama la atención que no haya un examen para las personas que siguen la asignatura con regularidad, que fundamentalmente se evalúa por un sistema de heteroevaluación y coevaluación, que se negocia en las primeras sesiones y se ajusta en las fechas a las necesidades del grupo-clase. También sorprende y llama su atención la propuesta de realizar una actividad en el entorno natural, el Parque Nacional de Monfragüe, de la que ellos se deben hacer responsables mediante una comisión.

También ha resultado muy interesante el conjunto de propuestas para realizar actividades de formación fuera del aula relacionadas con la asignatura, como el itinerario 
interpretativo por el Ecomuseo Miraflores, la web de la asignatura, jornadas de emprendimiento, laboratorio Ciudadanía, Patrimonio y Territorio (CIPAT), jornadas sobre memoria histórica, etc. Es importante resaltar que estos eventos han gustado mucho, aunque la participación en las jornadas y el laboratorio son puntuales por parte de pocos estudiantes.

El proyecto de investigación sirve como punto de arranque de la asignatura, tanto para el trabajo en grupo como para la construcción de conocimiento en ciencias sociales, otros años solo ha tenido la premisa de abordarse desde la perspectiva de conflictos socioambientales (Ruiz-Morales y Ferreras, 2018; Ruiz-Morales, 2018), pero este año se ha ampliado al vincularlos con los procesos de participación ciudadana. Esto responde a la idea de vincular la temática con una problemática de actualidad conectada con el territorio más próximo, buscando además que descubran el papel de la sociedad civil (entidades, movimientos sociales y procesos participativos). Para ello, a modo introductorio, se realizó la actividad 2.1 Aprendiendo a trabajar con problemas socioambientales. Análisis de conflictos y aportaciones del conocimiento científico a las ciencias sociales. Que los predispone a prestar atención a cuestiones socioambientales y desde una perspectiva más crítica y participativa.

Se ha seguido una estructura para presentar y dinamizar el proyecto de investigación, acorde con una visión científica del conocimiento en ciencias sociales, para ello se planteó la definición del problema socioambiental de investigación, la construcción de una trama de preguntas que sirvan como focos de atención sobre los que investigar, el enunciado de hipótesis, la definición de un contexto sobre el que realizar la investigación, el diseño de instrumentos, todo ello presentado en un pre-diseño.

Es importante resaltar la dificultad que entraña para la mayoría del grupo elaborar preguntas sugerentes, provocadoras, que movilicen para la investigación, porque 
habitualmente están muy acostumbrados a elaborar epígrafes, apartados, mapas conceptuales, etc.

En este momento se realizó la primera tutoría grupal, para ver la marcha de los grupos y cómo estaban abordando el pre-diseño, apoyar el trabajo de aquellos grupos que podían presentar alguna incidencia. En general todos los grupos marchaban muy bien, pero se veía diferencias importantes en el modo de organizar las tareas. De hecho la mayoría, un total de 6 de los 9 existentes, no había elaborado una planificación y tampoco había hecho un reparto de tareas. Este es una de las principales dificultades que presentan año a año los estudiantes en tercero, no tienen experiencias reales de trabajo en grupo, han realizado trabajos grupales pero simplemente repartiendo apartados del trabajo y después juntando el trabajo individual de cada uno. Carecen de un sentido y experiencia de trabajo colectivo, hay un grupo de estudiantes que perciben este tipo de trabajos como una carga, porque además prevén que sus compañeros no se van a implicar, por lo que intentan realizarlos con las mismas personas con las que saben que han funcionado bien otros años.

Esto además redunda en un desconocimiento de la mayoría del grupo clase, no se saben ni si quiera los nombres, por tanto no hay una identidad de grupo, sino sumas de individuos y subgrupos.

Las diferentes actividades que se han realizado para presentar a las personas del grupo clase, favorecer las interacciones, realizar actividades con personas distintas al grupo de trabajo estable, han sido valoradas muy positivamente por todas las personas. Tanto en el diario como en las sesiones destacan estas actividades, el proyecto de investigación y las actividades fuera del aula, porque habitualmente no las han realizado.

Al finalizar el proyecto de investigación han realizado un póster, para presentar los resultados del mismo y también han elaborado un dossier, que pretende ser como el material de consulta sobre el que construir la propuesta didáctica al final de la asignatura. 
A modo de evaluación, intentando constatar los cambios significativos que se han producido, centrándome en los problemas de investigación antes planteados, desearía realizar algunas precisiones en base a los datos recogidos en los diferentes instrumentos antes reseñados:

- ¿Cómo mejorar la implementación de los contenidos teóricos con la realización de actividades en el aula? Poseo algunas evidencias de que empezar por el proyecto de investigación, haciendo girar la lectura de los artículos y la elaboración de actividades de síntesis sobre este, ha generado un conjunto de aprendizajes más significativos. Según he podido comprobar en las tutorías grupales, preguntando a cada uno de los miembros del grupo, hay unanimidad de que este proyecto de investigación les ha permitido conocer la complejidad del mundo en el que viven, en más de un 50\% de los casos han manifestado leer de otro modo los medios de comunicación, porque han visto que existen intereses creados en el modo en el que ofrecen la información, han ampliado en general su conocimiento sobre las problemáticas investigadas, han aprendido a investigar de modo más riguroso y han aprendido a trabajar en grupo (ver figura 5). Si bien es cierto que el hecho de que cada asignatura genere un conjunto de actividades tan diverso y solapado en el tiempo, les genera mucha tensión. Esto acrecienta el nivel de exigencia en los grupos, lo que provoca conatos de conflicto que están relacionados habitualmente con la comunicación y la planificación de las actividades. De esto se han hecho más conscientes en la tutoría grupal.

- ¿De qué modo aprendemos a planificar y gestionar los tiempos para ser más eficientes en nuestra actividad profesional?

En la tutoría grupal y en el seguimiento realizado en algunas clases, se ha ido viendo la importancia de revisar los compromisos en el grupo, realizar y/o 
hacer seguimiento de la planificación. En ocasiones hay grupos que no saben identificar el conjunto de actividades que deben desarrollar, para llevar a buen puerto el proyecto de investigación, incluso a pesar de presentar con detalle las fases de la investigación. Llama la atención que en los diarios personales, casi no se toma como referencia el trabajo grupal, es como si el propio desarrollo del mismo no supusiese un aprendizaje en el día a día, más que cuando se les pregunta por la experiencia en tutoría. Esto está relacionado con las pocas experiencias que dicen tener todos en el aprendizaje y trabajo cooperativo (ver figura 5).

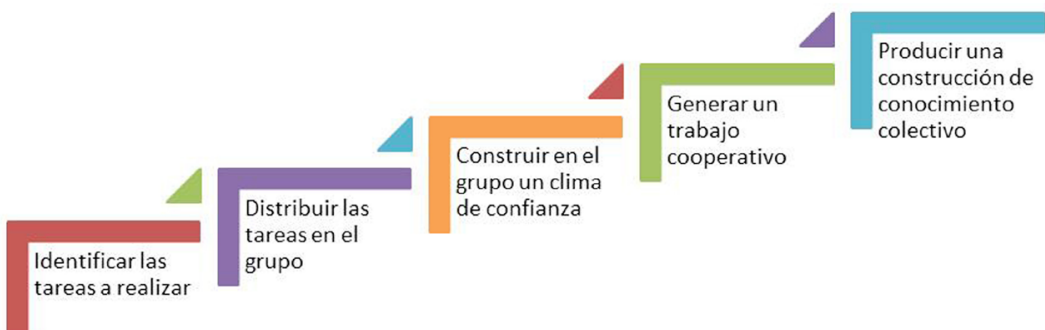

Figura 5. Escalera de conocimientos en el trabajo y aprendizaje cooperativo

- ¿Qué importancia tienen los procesos de participación ciudadana y el abordaje de los problemas socioambientales en las ciencias sociales? Han vivido de modo un tanto difícil el relacionar los conflictos socioambientales con otras problemáticas existentes que ellos ven como alejadas de consecuencias ambientales, caso de la inmigración, la precariedad laboral en la recogida de la fresa, el narcotráfico, etc. Fundamentalmente porque no integran en la sostenibilidad las dimensiones social, ambiental y económica. Además carecen de un pensamiento complejo que relacione acontecimientos sociales con repercusiones ambientales y viceversa, esto se constató en las primera tutoría grupal. En los trabajos nueve trabajos de investigación realizados solo el $50 \%$ ha 
dado un peso específico a las problemáticas socioambientales, casi más porque se han centrado en ellos que porque hayan realizado un trabajo que implique las tres dimensiones (ver figura 6). También llama la atención que utilizan a las entidades sociales como fuentes de información, pero no las consideran actores o sujetos, como parte del conflicto, lo que diluye su papel o importancia a la hora de tomarlos como elementos. Esto es algo a trabajar en el diseño de la propuesta didáctica, porque trabajar los problemas del mundo implica también abordarlos desde el papel de las instituciones y la sociedad civil, bien en su papel complementario o de

Identificar

problemas

socioambientales

Tomar como

referencia los

movimientos

sociales para

analizar las

problemáticas

Vincular los

problemas y los

procesos de

participación

ciudadana

Figura 6. Escalera de progresión problemas socioambientales y procesos de participación

- El subproblema ¿Cuáles son las ideas y concepciones del alumnado sobre nuestras problemáticas socioambientales? No ha podido ser analizado porque no se había entregado cuando se finalizó, y no ha sido posible tampoco su análisis.

El clima de trabajo ha sido positivo en cuanto a las actividades en grupo, sin embargo se ha detectado menos implicación y disponibilidad que otros años para realizar actividades como la relatoría, los estudiantes han ido mostrando en las sesiones sus reflexiones y en las entregas de las actividades demuestran mucho interés por profundizar. En las actividades cotidianas de los grupos que trabajan de modo permanente aparecen tensiones y algunos 
conflictos que son propios del proceso de aprendizaje de trabajar en grupo. También es un elemento de dificultad la falta de tiempo que aumenta el número de conflictos, así como la falta de herramientas para afrontar los conflictos desde un enfoque de comunicación no violenta. Todo ello motiva que año tras año, se convierta en uno de los grandes aprendizajes para los estudiantes en la asignatura.

En la línea de lo que suele ocurrir otros años, el análisis de la primera entrega del diario personal arroja resultados dispares, aproximadamente un $25 \%$ de los estudiantes realiza un diario donde toma como referencia la metodología de clase para reflexionar sobre su modelo didáctico, realiza reflexiones existenciales sobre el sentido de la educación y toma en consideración los contenidos de la asignatura, eventos que suceden en la vida cotidiana, tipo noticias, así como aportaciones de los compañeros. Mientras un $75 \%$ realiza un diario completamente descriptivo, sin mucha profundidad, esto se va invirtiendo en la entrega final que realizan en diciembre.

\section{Evaluación del CMD}

Entre las cuestiones a mantener en años sucesivos se encuentra seguir trabajando los contenidos de la asignatura a partir del proyecto de investigación sobre problemáticas socioambientales vinculadas con procesos de participación ciudadana y su presentación mediante un póster, también la indagación sobre las ideas y concepciones del alumnado de primaria, por último toda la dinámica de trabajos grupales que les permite aprender a organizarse. Considero fundamental reducir el número de actividades en relación con el proyecto de investigación, eliminando el dossier, que puede ser de poca ayuda de cara a la elaboración de la propuesta didáctica.

Es posible que para otro año, como parte del pre-diseño, se pida que presenten una planificación detallada de las actividades y tareas, de modo que solo falte después 
ponerle fechas y responsables, para que además puedan ubicar estas en las diferentes fases. Ayudándoles de este modo a la organización de las tareas.

El diario personal es un instrumento muy útil para conocer en cada momento como van reflexionando sobre los acontecimientos, en relación con los contenidos, cómo se sienten y construyen su modelo didáctico personal. En esta dirección creo que realizar dos sesiones de tutoría con cada grupo, también me sirve para conocer mejor a cada estudiante, el trabajo y asesorar.

Un principio didáctico fundamental es trabajar implicando a los estudiantes en las actividades de la asignatura, a partir de mostrarlas significativas, relevantes y atractivas, les permite "aprender desde el hacer" para aproximarse a los contenidos de la asignatura, esto me parece muy revelador y me ha dado buen resultado.

Considero crucial trabajar en la línea de coordinar esfuerzos entre profesorado que podamos compartir enfoque y modos de entender la formación, la docencia y la investigación para hacer más eficientes las actividades de clase, además de ofrecer un testimonio de coherencia entre lo que pregonamos como deseable y lo posible.

Jornadas de Formación e Innovación Docente del Profesorado | № 1 (2018) Esta obra se distribuye con la licencia Creative Commons 


\section{Referencias bibliográficas.}

Barchiesi, F., y otros (2010). La Universidad en conflicto. Capturas y fugas en el mercado global del saber. Navarra: Traficantes de sueños.

Ruiz-Morales, J. (2017a). ¿Qué importancia tiene trabajar el conocimiento de uno mismo y del grupo en la implementación de la didáctica de las ciencias sociales?. En Porlán, R. y Navarro, E. (Coordinan), Actas de las Jornadas de Formación e Innovación Docente (pp. 980-993). Sevilla: ICE.

Ruiz-Morales, j. (2017b): La voz de los estudiantes en el aprendizaje de las ciencias sociales. La construcción de espacios preparados para el aprendizaje. En Martínez, R., García-Morís, R. y García, C. R. (Editores), Investigación en didáctica de las ciencias sociales. Retos, preguntas y líneas de investigación (pp. 539-547).Córdoba: Universidad de Córdoba y Asociación de Profesorado de Didáctica en las Ciencias Sociales.

Ruiz-Morales, J. (2018). Trabajando a partir de conflictos socioambientales de nuestro mundo. Retos para la sostenibilidad curricular y oportunidades para la didáctica de las ciencias sociales. En López Torres, E., García Ruíz, C.R. y Sánchez Agustí, M., Buscando formas de enseñar: Investigar para innovar en Didáctica de las Ciencias Sociales (pp. 1061-1070). Valladolid: Universidad de Valladolid y Asociación Universitaria del Profesorado de Didáctica de las Ciencias Sociales. Recuperado de http://uvadoc.uva.es/handle/10324/28975

Ruiz-Morales, J. y Ferreras Listán, M. (2018). Los problemas socioambientales como motor de aprendizaje en la investigación escolar. En López Torres, E., García Ruíz, C.R. y Sănchez Agustí, M., Buscando formas de enseñar: Investigar para innovar en Didáctica de las Ciencias Sociales (pp. 457-468). Valladolid: Universidad de Valladolid y Asociación Universitaria del Profesorado de Didáctica de las Ciencias Sociales. Recuperado de http:// uvadoc.uva.es/handle/10324/28975 